\title{
Pressure dependence of diffusion coefficient and orientational relaxation time for acetonitrile and methanol in water: DRISM/mode-coupling study
}

\author{
A.E.Kobryn ${ }^{1}$, T. YAmaguchi $^{2}$ And F.Hirata ${ }^{1, *}$ \\ ${ }^{1}$ Institute for Molecular Science, Myodaiji, Okazaki, Aichi 444-8585, Japan \\ ${ }^{2}$ Department of Molecular Design and Engineering, Graduate School of Engineering, \\ Nagoya University, Chikusa, Nagoya, Aichi 464-8603, Japan
}

\begin{abstract}
We present results of theoretical description and numerical calculation of the dynamics of molecular liquids based on the Reference Interaction Site Model / Mode-Coupling Theory. They include the temperature-pressure(density) dependence of the translational diffusion coefficients and orientational relaxation times for acetonitrile and methanol in water at infinite dilution. Anomalous behavior, i.e. the increase in mobility with density, is observed for the orientational relaxation time of methanol, while acetonitrile does not show any deviations from the usual. This effect is in qualitative agreement with the recent data of MD simulation and with experimental measurements, which tells us that presented theory is a good candidate to explain such kind of anomalies from the microscopical point of view and with the connection to the structure of the molecules.
\end{abstract}

\section{Introduction}

Dynamics in solutions has been a central issue in the physical chemistry. It has been so because liquid dynamics is an important factor which determines the rate of chemical processes in solution including chemical reactions and protein folding. A variety of experimental methods have been devised to observe liquid dynamics, which now cover the dynamic range from femtoseconds to days.

Theoretical studies of liquid dynamics and relaxation have been dominated during the past century by the two continuum models which had been established essentially in the 18th century: the electrodynamics and the hydrodynamics [1]. The monumental achievements made in the field of liquid dynamics by the great scientists such as A. Einstein [2], P. Debye [3], L. Onsager [4,5] and M. Born [6] are essentially based on the continuum theories: the dielectric relaxation, the ion mobility and its concentration dependence, and so on. Such theories represented by the Stokes-Einstein-Debye (SED) law rely on the boundary conditions as well as phenomenological use of macroscopic constants such as the viscosity and dielectric constants in order to realize "chemistry" specific of the problem in concern. Typical examples of the boundary conditions are the "slip" and "stick" ones often employed in equations based on the hydrodynamic theory.

\footnotetext{
${ }^{*}$ To whom correspondence should be addressed. E-mail: hirata@ims.ac.jp
} 
The continuum models have played crucial roles in describing physics taking place in solution with the spatial as well as temporal resolutions which are low enough so that molecular details of the process can be neglected. There have been gaps between the phenomenological model and molecular processes. The gaps have been filled by using such adjectives as "effective". For example, the conductivity of a simple ion in aqueous solutions at infinite dilution depends on their size in entirely opposite manner to what is predicted from the Stokes law, if one employs the crystal radii as the ion size. In order to fill the gap between the phenomenological model and the molecular process, people have invented an "effective" radius or the Stokes radius, and interpreted the phenomenon in terms of the enhanced Stokes radii due to "hydration". So called "fractional exponent", e.g. [7], often used in the interpretation of the viscosity dependence of a relaxation rate in liquids is another example which tries to compromise the contradiction between phenomenological models and experiments. If an experimentalist tries to plot a relaxation rate against viscosity, he will often face serious violation of a law predicted by a phenomenological theory. It has been a common maneuver in such cases to use "fractional" dependence on viscosity. However, the use of "fractional" dependence does not add any information to describe molecular process actually taking place in solutions, but just hides the breakdown of the phenomenological theory. Recent experimental techniques, which are dramatically improved both in resolution and sensitivity, have demonstrated unambiguously breakdown of the phenomenological model. Let us refer to two contributions which exhibit such breakdown. Nakahara [8] and co-workers have shown that the rotational dynamics of a solute in a variety of solvents does not correlate with the viscosity of the solvent but rather with specific interactions between solute and solvent. Terazima and his coworkers [9] have found that the diffusion constant of radical species is roughly a half of that of their parent molecules, which are about the same size. We suppose no more words are necessary to convince people what may happen if one tries to describe more complicated dynamics in solution, e.g., conformational change of protein and chemical reactions, in terms of the phenomenological models.

Are there, then, any hope for theories of solution dynamics to break through the old regime established by the great scientists? We say "yes", if one relies on the two theories in the statistical mechanics developed in the last century: the generalized Langevin equation (GLE) [10] and the RISM theory of molecular liquids [11-13]. The generalized Langevin theory describes the time evolution of few dynamic variables in concern as a function of the representative point in the phase space. All other variables which are not under explicit concern are projected onto the dynamic variables of interest with the help of a projection operator. The projection leads to an equation which looks similar to the phenomenological Langevin equation containing the frictional force proportional to the rate of change of the dynamic variables, and the random force, which are related to each other by a fluctuation-dissipation theorem. If one chooses as the dynamic variables the density and the conjugated flux of sites or atoms of molecules in liquids, the theory gives an equation for the dynamic structure factor of atoms, which describes the time evolution of the site-site density pair correlation functions [14]. Results from the RISM theory, the site-site static structure factor and the direct correlation functions, are naturally plugged into GLE in order to evaluate not only the initial condition of the dynamics or the static structure factor, but also the frictional force as well as the collective frequency which 
concerns the frequency of the site-site density fluctuation. A crucial development of the theory is rather conceptual, not mathematical, in the sense that it has provided a new concept to view dynamics of a molecule in solution, which is quite different from the model traditionally exploited in the field. The new model sees the molecular motion in liquid as a correlated translational motion of atoms: if two atoms in a diatomic molecule are moving in the same direction, then the molecule as a whole is making a translational motion, while the molecule should be rotating if its atoms are moving in opposite directions. The view is different from the rotational-translational model traditionally developed based on the angular coordinates [15-17].

The new theory of liquid dynamics has been successfully applied to a variety of relaxation process in solution including the collective excitations in water [18], ion dynamics in dipolar liquids [19], dynamical Stokes shift [20,21], pressure dependence of the transport coefficients [22] as well as dielectric relaxation spectrum in water [23], and so forth. In the present proceeding, we report our latest study on dynamics of a molecule in solution at infinite dilution and its temperature and density dependence.

\section{Theory}

\section{Equilibrium structure}

In this work, we use the dielectrically-consistent reference interaction-site model (or DRISM for brevity) formalism [24] for the system. The main equation here is the site-site Ornstein-Zernike equation (SSOZ) written as

$\boldsymbol{\rho} \tilde{\mathbf{h}}(k) \boldsymbol{\rho}-\tilde{\boldsymbol{\chi}}(k)=[\tilde{\boldsymbol{\omega}}(k)+\tilde{\boldsymbol{\chi}}(k)] \tilde{\mathbf{c}}(k)[\tilde{\boldsymbol{\omega}}(k)+\tilde{\boldsymbol{\chi}}(k)]+[\tilde{\boldsymbol{\omega}}(k)+\tilde{\boldsymbol{\chi}}(k)] \tilde{\mathbf{c}}(k)[\boldsymbol{\rho} \tilde{\mathbf{h}}(k) \boldsymbol{\rho}-\tilde{\boldsymbol{\chi}}(k)]$,

where $\boldsymbol{\rho}$ is the diagonal matrix of number density of molecular species, and $\tilde{\mathbf{h}}(k), \tilde{\mathbf{c}}(k)$ and $\tilde{\boldsymbol{\omega}}(k)$ are the total, direct and intramolecular correlation matrices, respectively, in the reciprocal space. The matrix $\tilde{\chi}(k)$ is determined by the dielectric properties of the system.

In the case of infinite dilution limit, i.e. when concentration of one species (called a solute) tends to zero and concentration of other (called a solvent) essentially determines the total density, the DRISM equation can be decomposed as follows:

$\tilde{\mathbf{h}}^{\mathrm{vv}}(k)=\left[\tilde{\mathbf{w}}^{\mathrm{v}}(k)+\tilde{\mathbf{D}}^{\mathrm{v}}(k) \boldsymbol{\rho}^{\mathrm{v}}\right] \tilde{\mathbf{c}}^{\mathrm{vv}}(k)\left[\tilde{\mathbf{w}}^{\mathrm{v}}(k)+\boldsymbol{\rho}^{\mathrm{v}} \tilde{\mathbf{h}}^{\mathrm{vv}}(k)\right]+\tilde{\mathbf{D}}^{\mathrm{v}}(k)$,

$\tilde{\mathbf{h}}^{\mathrm{uv}}(k)=\tilde{\mathbf{w}}^{\mathrm{u}}(k) \tilde{\mathbf{c}}^{\mathrm{uv}}(k)\left[\tilde{\mathbf{w}}^{\mathrm{v}}(k)+\boldsymbol{\rho}^{\mathrm{v}} \tilde{\mathbf{h}}^{\mathrm{vv}}(k)\right]$,

$\tilde{\mathbf{h}}^{\mathrm{uu}}(k)=\tilde{\mathbf{w}}^{\mathrm{u}}(k) \tilde{\mathbf{c}}^{\mathrm{uu}}(k) \tilde{\mathbf{w}}^{\mathrm{u}}(k)+\tilde{\mathbf{w}}^{\mathrm{u}}(k) \tilde{\mathbf{c}}^{\mathrm{uv}}(k) \boldsymbol{\rho}^{\mathrm{v}} \tilde{\mathbf{h}}^{\mathrm{vu}}(k)$,

where $\tilde{\mathbf{w}}(k)=\tilde{\boldsymbol{\omega}}(k) \boldsymbol{\rho}^{-1}, \tilde{\mathbf{D}}(k)=\boldsymbol{\rho}^{-1} \tilde{\boldsymbol{\chi}}(k) \boldsymbol{\rho}^{-1}$, and superscripts "u" and "v" refer to "solute" and "solvent", respectively. Equations (2) are solved with the hypernetted chain (HNC) type of closure specified as

$g_{\alpha \gamma}(r)=\exp \left[-\frac{\phi_{\alpha \gamma}(r)}{k_{\mathrm{B}} T}+h_{\alpha \gamma}(r)-c_{\alpha \gamma}(r)\right]$,

where functions without tilde are those in the real space, $\alpha$ and $\gamma$ are site labels, and $g_{\alpha \gamma}(r) \equiv\left[h_{\alpha \gamma}(r)+1\right]$ is the site-site radial distribution function. Interaction potential between sites $\alpha$ and $\gamma$ is denoted as $\phi_{\alpha \gamma}(r), \beta=1 / k_{\mathrm{B}} T$ with $k_{\mathrm{B}}$ and $T$ being the Boltzmann constant and the absolute temperature, respectively. 


\section{The site-site mode-coupling theory}

The site-site intermediate scattering function of neat solvent and its self-parts are obtained by the generalized Langevin equation / modified mode-coupling theory for molecular liquids as is described in the previous study by Yamaguchi et al. [22]. Generalized Langevin equations for the neat solvent are given by

$$
\begin{array}{r}
\ddot{\mathbf{F}}(k, t)+k^{2} \mathbf{J}(k) \cdot \mathbf{S}^{-1}(k) \cdot \mathbf{F}(k, t)+\int_{0}^{t} \mathrm{~d} \tau \mathbf{K}(k, t-\tau) \cdot \dot{\mathbf{F}}(k, \tau)=\mathbf{0}, \\
\ddot{\mathbf{F}}^{\mathrm{s}}(k, t)+k^{2} \mathbf{J}(k) \cdot \mathbf{w}^{-1}(k) \cdot \mathbf{F}^{\mathrm{s}}(k, t)+\int_{0}^{t} \mathrm{~d} \tau \mathbf{K}^{\mathrm{s}}(k, t-\tau) \cdot \dot{\mathbf{F}}^{\mathrm{s}}(k, \tau)=\mathbf{0} .
\end{array}
$$

Here, the site-site intermediate scattering function and its self-part in the time domain, denoted as $\mathbf{F}(k, t)$ and $\mathbf{F}^{\mathrm{s}}(k, t)$, respectively, are given by

$$
\begin{aligned}
& F_{\alpha \gamma}(k, t) \equiv \frac{1}{N}\left\langle\rho_{\alpha}^{*}(k, t=0) \rho_{\gamma}(k, t)\right\rangle, \\
& F_{\alpha \gamma}^{\mathrm{s}}(k, t) \equiv \frac{1}{N}\left\langle\rho_{\alpha}^{*}(k, t=0) \rho_{\gamma}(k, t)\right\rangle^{\mathrm{s}},
\end{aligned}
$$

where $N$ is the number of solvent molecules, $\rho_{\alpha}(k)$ is the density field of $\alpha$-site in the reciprocal space, and $\langle\cdots\rangle$ means the statistical average, the suffix "s" means that correlations between the quantities of different molecules are neglected. Connection with equilibrium properties is expressed in the relation between the site-site static structure factor $\mathbf{S}(k) \equiv \mathbf{F}(k, t=0)$ and direct and intramolecular correlation functions, which is

$S_{\alpha \gamma}(k) \equiv \frac{1}{N}\left\langle\rho_{\alpha}^{*}(k, t=0) \rho_{\gamma}(k, t=0)\right\rangle=\left[\tilde{\mathbf{w}}^{\mathrm{v}}(k)+\rho \tilde{\mathbf{h}}^{\mathrm{vv}}(k)\right]_{\alpha \gamma}$.

The site-current correlation matrix $\mathbf{J}(k)$ is defined in a similar way as

$J_{\alpha \gamma}(k) \equiv \frac{1}{N}\left\langle j_{\alpha, z}^{*}(k, t=0) j_{\gamma, z}(k, t=0)\right\rangle$,

where $z$-axis is taken to be parallel to the $\mathbf{k}$ vector. The mode-coupling expressions of the memory function matrices, denoted as $\mathbf{K}(k, t)$ and $\mathbf{K}^{\mathrm{s}}(k, t)$, are given by

$$
\begin{aligned}
{\left[\mathbf{J}^{-1}(k) \cdot \mathbf{K}_{\mathrm{MCT}}(k, t)\right]_{\alpha \gamma}=} & \frac{\rho}{(2 \pi)^{3}} \int \mathrm{d} \mathbf{q}\left\{q_{z}^{2}\lfloor\tilde{\mathbf{c}}(q) \cdot \mathbf{F}(q, t) \cdot \tilde{\mathbf{c}}(q)\rfloor_{\alpha \gamma} \mathbf{F}_{\alpha \gamma}(|\mathbf{k}-\mathbf{q}|, t)\right. \\
& \left.-q_{z}\left(k-q_{z}\right)\lfloor\tilde{\mathbf{c}}(q) \cdot \mathbf{F}(q, t)\rfloor_{\alpha \gamma}\lfloor\mathbf{F}(|\mathbf{k}-\mathbf{q}|, t) \cdot \tilde{\mathbf{c}}(|\mathbf{k}-\mathbf{q}|)\rfloor_{\alpha \gamma}\right\}, \\
{\left[\mathbf{J}^{-1}(k) \cdot \mathbf{K}_{\mathrm{MCT}}^{\mathrm{s}}(k, t)\right]_{\alpha \gamma}=} & \frac{\rho}{(2 \pi)^{3}} \int \mathrm{d} \mathbf{q} q_{z}^{2}\lfloor\tilde{\mathbf{c}}(q) \cdot \mathbf{F}(q, t) \cdot \tilde{\mathbf{c}}(q)\rfloor_{\alpha \gamma} \mathbf{F}_{\alpha \gamma}^{\mathrm{s}}(|\mathbf{k}-\mathbf{q}|, t) .
\end{aligned}
$$

According to the recipe by Yamaguchi and Hirata [25], memory functions for the self-part are given by the linear combination of the corresponding mode-coupling memory functions as

$$
\begin{aligned}
& {\left[\mathbf{K}^{\mathrm{s}}(k, t) \cdot \mathbf{J}(k)\right]_{\alpha \gamma}=\sum_{\substack{m_{1,2,3} \in\{x, y, z\} \\
\mu, \nu \in i}}\left\langle u_{z m_{1}}^{(i)} Z_{m_{1} m_{2}}^{\alpha \mu} Z_{m_{2} m_{3}}^{\nu \gamma} u_{z m_{3}}^{(i)} \mathrm{e}^{i \mathbf{k} \cdot\left(\mathbf{r}_{i}^{\alpha}-\mathbf{r}_{i}^{\mu}-\mathbf{r}_{i}^{\gamma}+\mathbf{r}_{i}^{\nu}\right)}\right\rangle } \\
& \times\left[\mathbf{J}^{-1}(k) \cdot \mathbf{K}_{\mathrm{MCT}}^{\mathrm{s}}(k, t)\right]_{\alpha \gamma},
\end{aligned}
$$


where $Z_{m_{1} m_{2}}^{\alpha \gamma}$ and $u_{z m}^{(i)}$ stand for the orientation-dependent site-site velocity correlation matrix and the unitary matrix that describes the rotation between the molecular- and laboratory-fixed coordinates of molecule $i$, respectively [25]. The collective-part of the memory function (neglecting the orientational correlation between different molecules) is given by

$$
\mathbf{K}(k, t)=\mathbf{K}_{\mathrm{MCT}}(k, t)+\mathbf{K}^{\mathrm{s}}(k, t)-\mathbf{K}_{\mathrm{MCT}}^{\mathrm{s}}(k, t) .
$$

The time-evolution of the self-part of the solute-solute site-site intermediate scattering function is governed by the equations similar to Eqs. (4b), (8b) and (9) as

$$
\begin{aligned}
\ddot{\mathbf{F}}^{\mathrm{uu}, \mathrm{s}}(k, t)+k^{2} \mathbf{J}^{\mathrm{u}}(k) \cdot \mathbf{w}^{\mathrm{u},-1}(k) \cdot \mathbf{F}^{\mathrm{uu}, \mathrm{s}}(k, t)+\int_{0}^{t} \mathrm{~d} \tau \mathbf{K}^{\mathrm{uu}, \mathrm{s}}(k, t-\tau) \cdot \dot{\mathbf{F}}^{\mathrm{uu}, \mathrm{s}}(k, \tau)=\mathbf{0}, \\
{\left[\mathbf{J}^{\mathrm{uu},-1}(k) \cdot \mathbf{K}_{\mathrm{MCT}}^{\mathrm{uu}, \mathrm{s}}(k, t)\right]_{\alpha \gamma}=\frac{\rho}{(2 \pi)^{3}} \int \mathrm{d} \mathbf{q} q_{z}^{2}\left\lfloor\tilde{\mathbf{c}}^{\mathrm{uv}}(q) \cdot \mathbf{F}^{\mathrm{vv}}(q, t) \cdot \tilde{\mathbf{c}}^{\mathrm{vu}}(q)\right\rfloor_{\alpha \gamma} \mathbf{F}_{\alpha \gamma}^{\mathrm{uu}, \mathrm{s}}(|\mathbf{k}-\mathbf{q}|, t), } \\
{\left[\mathbf{K}^{\mathrm{uu}, \mathrm{s}}(k, t) \cdot \mathbf{J}^{\mathrm{uu}}(k)\right]_{\alpha \gamma}=\sum_{\substack{m_{1,2,3} \in\{x, y, z\} \\
\mu, \nu \in i}}\left\langle u_{z m_{1}}^{(i)} Z_{m_{1} m_{2}}^{\alpha \mu} Z_{m_{2} m_{3}}^{\nu \gamma} u_{z m_{3}}^{(i)} \mathrm{e}^{i \mathbf{k} \cdot\left(\mathbf{r}_{i}^{\alpha}-\mathbf{r}_{i}^{\mu}-\mathbf{r}_{i}^{\gamma}+\mathbf{r}_{i}^{\nu}\right)}\right\rangle } \\
\times\left[\mathbf{J}^{\mathrm{uu},-1}(k) \cdot \mathbf{K}_{\mathrm{MCT}}^{\mathrm{uu}, \mathrm{s}}(k, t)\right] .
\end{aligned}
$$

\section{Diffusion coefficient and reorientational relaxation time}

Based on the Green-Kubo formula, the translational diffusion coefficient $D$ is obtained as $[26,27]$

$D=\frac{1}{3} \int_{0}^{\infty} \mathrm{d} t Z_{\alpha \gamma}(t)$,

where $\mathbf{Z}(t)$ is the site-site velocity autocorrelation function, which is described in terms of the self-part of the intermediate scattering function $\mathbf{F}^{\mathrm{s}}(k, t)$ as

$Z_{\alpha \gamma}(t) \equiv \frac{1}{N} \sum_{i}\left\langle\mathbf{v}_{\alpha}(0) \cdot \mathbf{v}_{\gamma}(t)\right\rangle^{\mathrm{s}}=-\lim _{k \rightarrow 0} \frac{3}{k^{2}} \ddot{F}_{\alpha \gamma}^{\mathrm{s}}(k, t)$.

In Eq. (14), $\alpha$ and $\gamma$ refer to any two sites in a molecule which are bound by chemical bonds. The expression for the single-particle reorientational time is also described in terms of the site-site velocity autocorrelation function $[22,27,28]$. In this work, we restrict our discussion to the rank-1 reorientational relaxation of the dipole moment $\boldsymbol{\mu}$ given by

$\boldsymbol{\mu}_{i}=\sum_{\alpha} z_{\alpha} \mathbf{r}_{i}^{\alpha}$,

where $z_{\alpha}$ is the charge of the site $\alpha$. The first-rank reorientational correlation function $C_{\boldsymbol{\mu}}(t)$ is defined as

$C_{\boldsymbol{\mu}}(t) \equiv \frac{\sum_{i}\left\langle\boldsymbol{\mu}_{i}(0) \boldsymbol{\mu}_{i}(t)\right\rangle}{\sum_{i}\left\langle\left|\boldsymbol{\mu}_{i}^{2}\right|\right\rangle}$. 
Substituting Eq. (16) into Eq. (17),$C_{\boldsymbol{\mu}}(t)$ is related to the site-site velocity autocorrelation function $\mathbf{Z}(t)$ as

$C_{\boldsymbol{\mu}}(t)=\frac{\sum_{i} \sum_{\alpha \gamma} z_{\alpha} z_{\gamma}\left\langle\mathbf{r}_{i}^{\alpha}(t) \mathbf{r}_{i}^{\gamma}(0)\right\rangle}{\sum_{i}\left\langle\left|\boldsymbol{\mu}_{i}^{2}\right|\right\rangle}, \quad \ddot{C}_{\boldsymbol{\mu}}(t)=-\frac{N \sum_{\alpha \gamma} z_{\alpha} z_{\gamma} Z^{\alpha \gamma}(t)}{\sum_{i}\left\langle\left|\boldsymbol{\mu}_{i}^{2}\right|\right\rangle}$.

The reorientational correlation time of the 1 st $\operatorname{rank}, \tau$, is then defined as the timeintegration of $C_{\boldsymbol{\mu}}(t)$.

\section{Description of the models}

We performed explicit calculations for two popular systems, i.e. acetonitrile $\left(\mathrm{CH}_{3} \mathrm{CN}\right)$ in water and methanol $\left(\mathrm{CH}_{3} \mathrm{OH}\right)$ in water, both in the case of infinite dilution. As for the structure and the intermolecular potential of water we employed a model of the extended simple point charge (SPC/E) [29]. We also put the Lennard-Jones (LJ) core on the hydrogen atoms in order to avoid the undesired divergence of the solution of the RISM integral equation. The LJ parameters of the hydrogen atom, the depth of the well and the diameter, are chosen to be $0.046 \mathrm{kcal} / \mathrm{mol}$ and $0.7 \AA$, respectively.

In acetonitrile and methanol the methyl group is considered to be a single interaction site (Me) located on the methyl carbon atom. So that both chemical compounds consist of three sites interacting through the pair potential $[30,31]$

$\phi\left(r_{i}, r_{j}\right) \equiv \phi\left(r_{i j}\right)=\sum_{\alpha, \beta}^{3}\left\{4 \epsilon_{\alpha \beta}\left[\left(\frac{\sigma_{\alpha \beta}}{r_{i \alpha, j \beta}}\right)^{12}-\left(\frac{\sigma_{\alpha \beta}}{r_{i \alpha, j \beta}}\right)^{6}\right]+\frac{z_{\alpha} z_{\beta}}{r_{i \alpha, j \beta}}\right\}$,

i.e., LJ plus Coulomb. Here $\alpha$ and $\beta$ label sites on molecules $i$ and $j ; r_{i \alpha, j \beta}=\left|\mathbf{r}_{i \alpha}-\mathbf{r}_{j \beta}\right|$; parameters $\epsilon_{\alpha \beta}$ and $\sigma_{\alpha \beta}$ are LJ well-depths and LJ diameters defined as $\epsilon_{\alpha \beta}=\sqrt{\epsilon_{\alpha} \epsilon_{\beta}}$ and $\sigma_{\alpha \beta}=\left(\sigma_{\alpha}+\sigma_{\beta}\right) / 2$, respectively, with $\sigma_{\alpha}$ being the LJ diameter of a single site. Point charges for acetonitrile were chosen to reproduce electrostatic potential obtained in an $a b$ initio calculations [30]. Numerical values of parameters of the site-site interaction potential (19) and masses of sites are specified in Table 1

Table 1: Parameters of the site-site interaction potential (19): mass and charge are in atomic units, $\sigma_{\mathrm{LJ}}$ in $\AA$, and $\epsilon_{\mathrm{LJ}}$ in $10^{-14} \mathrm{erg} / \mathrm{molec}$.

\begin{tabular}{cccccccccc} 
& \multicolumn{3}{c}{ water $^{\mathrm{a}}$} & \multicolumn{3}{c}{ acetonitrile $^{\mathrm{b}}$} & \multicolumn{3}{c}{ methanol $^{\mathrm{c}}$} \\
& \multicolumn{1}{c}{$\mathrm{O}$} & $\mathrm{H}_{1}$ & $\mathrm{H}_{2}$ & $\mathrm{Me}$ & $\mathrm{C}$ & $\mathrm{N}$ & $\mathrm{Me}$ & $\mathrm{O}$ & $\mathrm{H}$ \\
\hline$m$ & 16.0 & 1.008 & 1.008 & 15.024 & 12.0 & 14.0 & 15.024 & 16.0 & 1.008 \\
$z$ & -0.8476 & 0.4238 & 0.4238 & 0.269 & 0.129 & -0.398 & 0.265 & -0.7 & 0.435 \\
$\sigma_{\mathrm{LJ}}$ & 3.16 & 0.7 & 0.7 & 3.6 & 3.4 & 3.3 & 3.74 & 3.03 & 1.0 \\
$\epsilon_{\mathrm{LJ}}$ & 1.084 & 0.3196 & 0.3196 & 2.64 & 0.6878 & 0.6878 & 1.4525 & 1.1943 & 0.3196 \\
\hline \multicolumn{3}{r}{${ }^{2}$ Ref. $[29]$} & \multicolumn{3}{r}{ Ref. [30] } & \multicolumn{3}{c}{${ }^{c}$ Ref. [31] }
\end{tabular}

Information about bond length can be deduced from Cartesian coordinates $(x, y, z)$ of sites indicated in Table 2 (principal axes and the origin can be taken arbitrarily). In calculations, the temperature of the system is varied from 258.15 to $373.15 \mathrm{~K}$, and the number-density from 0.03008 to 0.04011 molecules $/ \AA^{3}$, where the number-density of water 
Table 2: Cartesian coordinates of sites.

\begin{tabular}{|c|c|c|c|c|c|}
\hline \multicolumn{2}{|r|}{ water } & \multicolumn{2}{|c|}{ acetonitrile } & \multicolumn{2}{|r|}{ methanol } \\
\hline site & $(x, y, z), \bar{A}$ & site & $(x, y, z), \bar{A}$ & site & $(x, y, z), \AA$ \\
\hline $\mathrm{O}$ & $(0,0 \quad,-0.0646)$ & $\mathrm{Me}$ & $(0,0,1.46)$ & $\mathrm{Me}$ & $(-1.4246,0$ \\
\hline $\mathrm{H}_{1}$ & $(0,0.8165,0.5127)$ & $\mathrm{C}$ & $(0,0,0 \quad)$ & $\mathrm{O}$ & $\left(\begin{array}{ll}0 & , 0\end{array}\right.$ \\
\hline $\mathrm{H}_{2}$ & $(0,-0.8165,0.5127)$ & $\mathrm{N}$ & $(0,0,-1.17)$ & $\mathrm{H}$ & $(0.3004,0.8961,0)$ \\
\hline
\end{tabular}

at the ambient conditions is 0.03334 molecules $/ \AA^{3}$. Connection of these parameters with thermodynamic pressure is shown in Table 3 (except for the metastable regions where we do not have reliable data). Temperature/density dependent dielectric permittivity $\varepsilon$

Table 3: Density-pressure correspondence for water according to [32]. Pressure is given in MPa.

\begin{tabular}{ccccc}
$\rho, \mathrm{g} / \mathrm{cm}^{3}$ & $n, \AA^{-3}$ & $273.15 \mathrm{~K}$ & $298.15 \mathrm{~K}$ & $373.15 \mathrm{~K}$ \\
\hline 0.9 & 0.03008 & - & - & - \\
1.0 & 0.03334 & 0.4085 & 6.6914 & 100.64 \\
1.1 & 0.03676 & 257.20 & 296.20 & 451.51 \\
1.2 & 0.04011 & 689.03 & 760.76 & 993.38 \\
\hline
\end{tabular}

for water used in numerical calculations has been evaluated as a physical solution of an empirical nonlinear equation presented in [33]:

$\varepsilon-\frac{1}{2}\left(1+\frac{1}{\varepsilon}\right)=\frac{1}{v}\left(17+\frac{9.32 \cdot 10^{4}\left(1+\frac{153}{v \cdot T^{1 / 4}}\right)}{\left(1-\frac{3}{v}\right)^{2} T}\right)$,

where $v$ is a molar volume in units of $\mathrm{cm}^{3} / \mathrm{mol}$, and $T$ is thermodynamic temperature in $\mathrm{K}$. This equation was also used in such temperature/density points where no experimental data exist.

\section{Numerical methods}

From the generalized Langevin equation / modified mode-coupling theory and the DRISM /HNC integral equation theory, the diffusion coefficients and the reorientational relaxation times of solute molecules in solution can be obtained based solely on the information about molecular shapes, inertia parameters, intermolecular interaction potentials, temperature and density. First, we calculated the site-site static structure factor by the DRISM/HNC equation using the intermolecular interaction, molecular shape, temperature and density. In order to improve the convergence of the RISM calculation, we used the method of the modified direct inversion in an iterative space proposed by Kovalenko et al. [34]. From the static site-site structure factor, we calculated the site-site intermediate scattering function using the site-site generalized Langevin equation / modified mode-coupling theory. The generalized Langevin equation is time-integrated numerically. The time-development of the correlation functions in the $k \rightarrow 0$ limit is separately treated by the analytical limiting procedure of the theoretical expressions. In the numerical procedure, the reciprocal space is linearly discretized as $k=\left(n+\frac{1}{2}\right) \Delta k$, where $n$ is the integer from 0 to $N_{k}-1$. Values of $\Delta k$ and $N_{k}$ are $0.061 \AA^{-1}$ and 512 , respectively. The diffusion coefficient $D$ was calculated 
from the asymptotic slope of the time dependence of the mean square displacement and the orientational relaxation time $\tau$ was analyzed using the rotational autocorrelation functions.

\section{Results and discussion}

Figure 1 shows the density dependence of normalized diffusion coefficients $D / D_{0}$, with $D_{0}$ being the diffusion coefficient at the lowest density, for both acetonitrile (left part) and methanol (right part) at four temperatures. Corresponding results for the orientational relaxation time $\tau$ are shown in figure 2. It has been found that diffusion coefficients of both acetonitrile and methanol at these temperatures decrease monotonically with increase of density except for methanol at the lowest temperature, where one observes a typical anomalous behavior: first diffusion coefficient increases, then decreases again. At the same time, behavior of orientational relaxation time is different for acetonitrile and methanol at all studied temperatures, except at the highest. One can easily observe that $\tau$ for acetonitrile exhibits the monotonic increase with density and monotonic decrease of its absolute value with temperature [figure 2(a)], while $\tau$ for methanol at the same temperatures first decreases with density and then increases [figure 2(c)]. Its absolute value is also getting smaller with temperature as in the case of acetonitrile. In such a way, the density dependent orientational relaxation time for dissolved in water methanol has an absolute minimum somewhere in between $0.9 \mathrm{~g} / \mathrm{cm}^{3}$ and $1.1 \mathrm{~g} / \mathrm{cm}^{3}$ for water and the depth of this minimum is seen more clearly at lower temperatures. These tendencies are observed sharply in figures 2(b) and 2(d) for acetonitrile and methanol, respectively, where we plot normalized values of $\tau$ as $\tau / \tau_{0}$ with $\tau_{0}$ being the orientational relaxation time at the lowest density. Obtained results are in qualitative agreement with the outco-
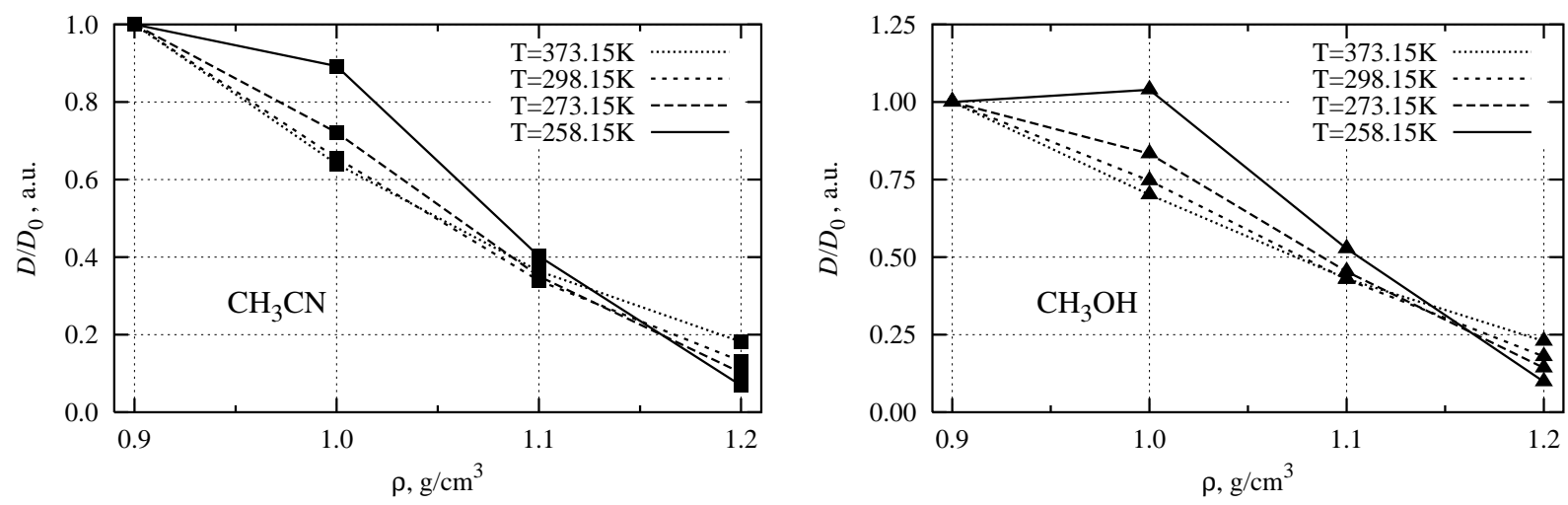

Figure 1: Density dependence of diffusion coefficients for acetonitrile (- $)$ and methanol ( $\mathbf{\Delta})$ in water at different temperatures.

me of the MD simulation by Chowdhuri and Chandra [35] performed for similar systems at $258 \mathrm{~K}$ and $298 \mathrm{~K}$, and with experimental measurements by Wakai and Nakahara [8] who observed likewise behavior for several different systems, including acetonitrile, at room temperatures.

The difference in behavior of acetonitrile and methanol can be made clear from the point of view of their molecular structure. To begin with, let us remind that the anomalous density dependence of the molecular mobility in water has been explained by Yamaguchi 

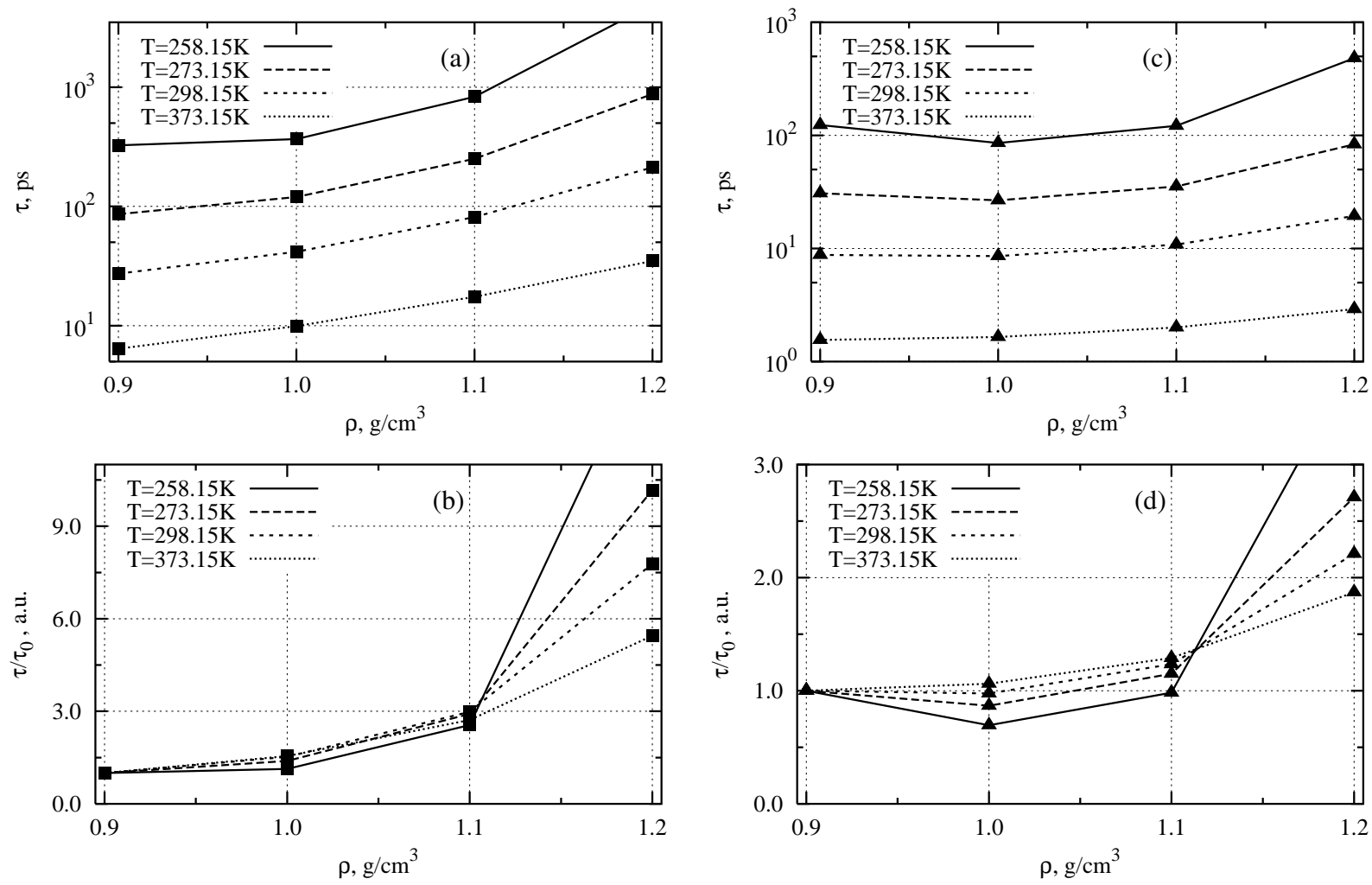

Figure 2: Density dependence of the orientational relaxation times for acetonitrile (-) and methanol $(\mathbf{\Lambda})$ in water at different temperatures.

et.al. [22] based on the facts of an almost spherical repulsive core for water and the strong short-range intermolecular Coulomb interaction called "hydrogen bonding". Following the idea and owing to the models used in present manuscript, one can conclude that since acetonitrile cannot make the hydrogen bonding and methanol does, this fact may be a good candidate to serve as a reason or cause of justification of the anomalous density behavior of methanol (or possibly any protic solution) and usual one of acetonitrile (or possibly any aprotic solution).

\section{Summary}

In present work we have calculated the temperature/density dependence of the translational diffusion coefficients and rank-1 orientational relaxation times for acetonitrile and methanol in water at infinite dilution using the site-site generalized Langevin equation / modified mode-coupling theory and the DRISM/HNC theory. Calculations show anomalous density dependence of the orientational relaxation time for methanol in water which is consistent with the results of experimental observation and MD simulation. On the other hand, similar computation for acetonitrile does not exhibit deviation from the usual behavior which is also in agreement with experiment and MD simulation. Such a difference is explained based on the molecular structure of solvents, in particular their ability (for methanol) and inability (for acetonitrile) to create hydrogen bonds. More studies will be required for better understanding of the molecular dynamics in such systems. 


\section{REFERENCES}

[1] G.G. Stokes, Trans. Camb. Phil. Soc. 9 (1850) 8.

[2] A. Einstein, Investigation on the Theory of the Brownian Movement, R. Fruth, ed., A.D. Cowper, transl. (Dover, New York, 1956).

[3] P. Debye, Polar Molecules (Chemical Catalog Company, New York, 1929).

[4] L. Onsager, J. Am. Chem. Soc. 58 (1936) 1486.

[5] J.B. Hubbard and L. Onsager, J. Chem. Phys. 67 (1977) 4850; J.B. Hubbard, J. Chem. Phys. 68 (1978) 1649.

[6] M. Born, Z. Phys. 1 (1920) 221.

[7] R. Zwanzig and A.K. Harrison, J. Chem. Phys. 83 (1985) 5861.

[8] C. Wakai and M. Nakahara, J. Chem. Phys. 100 (1994) 8347; ibid. 106 (1997) 7512.

[9] M. Terazima and N. Hirota, J. Chem. Phys. 95 (1991) 6490;

M. Terazima, K. Okamoto, and N. Hirota, J. Phys. Chem. 97 (1993) 13387.

[10] J.P. Hansen and I.R. McDonald, Theory of Simple Liquids (Academic Press, New York, 1986).

[11] D. Chandler and H.C. Andersen, J. Chem. Phys. 57 (1972) 1930.

[12] D. Chandler, in The Liquid State of Matter, ed. by E. Montroll and J.L. Lebowitz (North Holland, Amsterdam, 1982).

[13] F. Hirata and P.J. Rossky, Chem. Phys. Lett. 83 (1981) 329; F. Hirata, B.M. Pettitt, and P.J. Rossky, J. Chem. Phys. 77 (1982) 509; F. Hirata, P.J. Rossky, and B.M. Pettitt, J. Chem. Phys. 78 (1983) 4133.

[14] F. Hirata, J. Chem. Phys. 96 (1992) 4519.

[15] D.F. Calef and P.G. Wolynes, J. Chem. Phys. 78 (1983) 4145.

[16] A. Chandra and B. Bagchi, Chem. Phys. Lett. 151 (1988) 47; J. Chem. Phys. 90 (1989) 1832, 7338.

[17] D. Wei and G.N. Patey, J. Chem. Phys. 91 (1989) 7113; ibid. 93 (1990) 1399.

[18] S.-H. Chong and F. Hirata, J. Chem. Phys. 111 (1999) 3083, 3095.

[19] S.-H. Chong and F. Hirata, J. Chem. Phys. 111 (1999) 3654.

[20] F. Hirata, T. Munakata, F. Raineri, and H.L. Friedman, J. Mol. Liquids 65/66 (1995) 15.

[21] K. Nishiyama, F. Hirata, and T. Okada, J. Chem. Phys. 118 (2003) 2279.

[22] T. Yamaguchi, S.-H. Chong, and F. Hirata, J. Chem. Phys. 119 (2003) 1021.

[23] T. Yamaguchi, S-H. Chong, and F. Hirata, Mol. Phys. 101 (2003) 1211.

[24] J. Perkyns and M.B. Pettitt, Chem. Phys. Lett. 190 (1992) 626; J. Chem. Phys. 97 (1992) 7656.

[25] T. Yamaguchi and F. Hirata J. Chem. Phys. 117 (2002) 2216.

[26] S.-H. Chong and F. Hirata, Phys. Rev. E 57 (1998) 1691.

[27] S.-H. Chong, W. Götze and M.R. Mayr, Phys. Rev. E 64 (2001) 011503.

[28] T. Yamaguchi, S.-H. Chong and F. Hirata, J. Mol. Liquids 112 (2004) 117.

[29] H.J.J.C. Berendsen, J.R. Grigera and T.P. Straatsma, J. Phys. Chem. 91 (1987) 6269.

[30] D.M. Edwards, P.A. Madden and I.R. McDonald, Mol. Phys. 51 (1984) 1141.

[31] W.L. Jorgensen, J. Phys. Chem. 90 (1986) 1276.

[32] W. Wagner and A. Pruß, J. Chem. Phys. Ref. Data 31 (2002) 387.

[33] Landolt-Börnstein Numerical Data and Functional Relationships in Science and Technology, New Series, ed. in chief K.-H. Hellwege. Group IV: Macroscopic and Technical Properties of Matter. Vol. 4: High-Pressure Properties of Matter. G. Beggerow. Ed. Kl. Schäfer (Springer-Verlag, Berlin, 1980).

[34] A. Kovalenko, S. Ten-no and F. Hirata, J. Comp. Chem. 20 (1999) 928.

[35] S. Chowdhuri and A. Chandra, Chem. Phys. Lett. 373 (2003) 79. 